\title{
Seneca Valley Virus-001
}

National Cancer Institute

\section{Source}

National Cancer Institute. Seneca Valley Virus-001. NCI Thesaurus. Code C62524.

A replication-competent oncolytic picornavirus with potential antineoplastic activity.

Administered systemically, Seneca Valley virus-001 specifically targets and infects tumor

cells with neuroendocrine characteristics. Upon infection, this agent replicates

intracellularly, resulting in tumor cell lysis and reduced tumor cell proliferation. The

selective tropism of virus replication may involve receptor-mediated internalization. 